\title{
Fast Flooding in Power Save Mode of IEEE 802.11 DCF Based Mobile Ad Hoc Networks
}

\author{
Myung-Hwan Seo ${ }^{1}$, Hyeon-Ju Yoon ${ }^{2}$, and Joong-Soo $\mathrm{Ma}^{1}$ \\ 1 Information and Communications University, \\ 103-6, Munji-Dong, Yusong-Gu, Daejeon 305-714, S. Korea \\ http://mm.icu.ac.kr \\ 2 Korea Advanced Institute of Science and Technology, \\ 373-1, Gusong-Dong, Yusong-Gu, Daejeon 305-701, S. Korea \\ \{mhseo, jsma\}@icu.ac.kr, juyoon@kaist.ac.kr
}

\begin{abstract}
We propose Fast Flooding in Power Save Mode (FFPSM), a modification of IEEE 802.11 power save mode operation for Mobile Ad hoc NETworks (MANET). The previous works for the power save mode operation usually focused on shortening the duration of the awake state to minimize the power consumption of mobile nodes. We observed that the longer sleeping period results in seriously long delivery latency because the packets can move forward only one hop for a fixed interval. Our FFPSM scheme allows the flooding packets to be forwarded several hops in a transmission period. Each node propagates the announcements for next flooding to nodes of several hops away, thus the packets can travel multiple hops during one interval. Simulation results show that the proposed approach significantly reduces the end-to-end delay of flooding without extra energy consumption.
\end{abstract}

\section{Introduction}

Mobile ad hoc network (MANET) is a network of self-organized, wireless, mobile hosts with limited capacity of battery power. In the literature of MANET research, energy efficient techniques have been very important issues. IEEE 802.11 DCF (Distributed Coordination Function) [1] is a fully distributed MAC protocol for MANETs. It specifies a power saving mechanism, PSM (Power Save Mode), which consists of 3 states of power consumption, off, awake, and doze state. Very little power is consumed in the doze state in which the wireless interface is not able to transmit or receive.

In $\mathrm{DCF}$, time is divided into fixed intervals, beacon intervals $(B I)$, and each $B I$ is divided into ATIM (Ad hoc traffic Indication Message) window and data transmission window (Fig. 1). During the ATIM Window, each node wakes up and exchanges the announcements for next data transmission with neighbors. The nodes that send or receive ATIMs stay awake for data transmission and others go into the doze state. The data packets usually move forward hop by hop, taking one $B I$ per hop. 


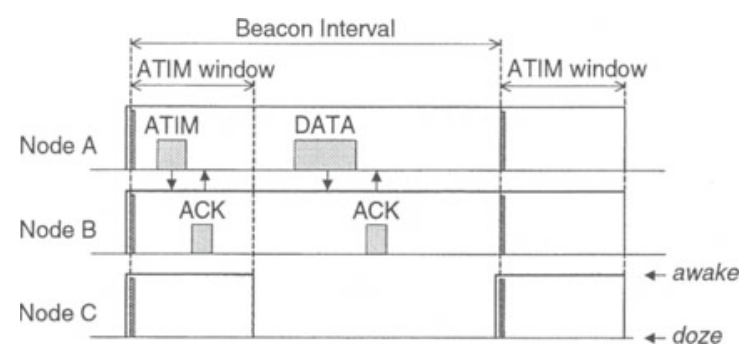

Fig. 1. Operation in IEEE 802.11 DCF Power Save Mode

Some modification of PSM have been developed to achieve more energy saving, by reducing the awake interval. However, the packet delivery latency from source to destination has not been an important issue, though it may generate significant user inconvenience and even more power consumption overhead.

We propose a new approach, FFPSM (Fast Flooding in Power Save Mode), which can significantly reduce the end-to-end delay of flooding and maintain other performance measures at the acceptable levels. The main idea is to maximize the utilization of the compulsory awake state interval, ATIM Window, not to shrink or adjust its size. FFPSM makes many nodes awake at the same time, so the packet flooding speed can be enhanced.

\section{Related Works}

In the IEEE 802.11 DCF-PSM, the different sizes of BI and ATIM Window have significant impact on energy saving and throughput achieved by the nodes. [2] recommended $95 \mathrm{~ms}$ of $B I$ and about $25 \%$ of $B I$ as $A T I M$ Window size.

DPSM (Dynamic PSM) by Jung et al.[3] dynamically adjusts the ATIM window size based on observed network conditions. It improved energy efficiency without degrading throughput, and they revealed that highly loaded network may cause low throughput per energy unit because of the extra use of channel capacity for ATIM Window. TIPS (Traffic Indication-based Power Saving)[4] is another approach to prolong the doze state. It allows the beacon transmission to indicate the presence of following ATIM, thus nodes to go to sleep early. Both [3] and [4], the modifications of IEEE 802.11 DCF-PSM, have focused on reducing the duration of the awake state, thus achieving more energy saving. However, they didn't pay attention to the long latency caused by the periodic sleeping and intermittent packet forwarding, and the effect of multi-hop transmission.

Span [5] is a power saving technique improving the latency. In span, only a small number of nodes called coordinator make a forwarding backbone and remain awake at any time to forward traffic for active connections, and others work in PSM. Coordinators are elected based on local decision and change periodically. With the geographic routing which uses excessive broadcasts, Span showed more energy saving and shorter latency than those of IEEE 802.11 PSM. 
However, it is customized for geographic routing, so the distributed coordinator election overhead would be large and usually intensive use of specific nodes may result in shorter network lifetime under other conditions.

On the other hand, some power saving techniques focused on the clock synchronization[6][7]. In the standard, nodes synchronize their clocks by distributed method. In real environment, due to the mobility and the radio interference, there is possibility not to recognize each other for a long time even though they are within the communication range. [6] and [7] proposed asynchronous PSM, in which each node repeats awake and doze state by a specific pattern devised to meet others without fail. However, packet delivery rate may degrade because of the asynchrouns and fewer coincidence, and become more critical in the case of broadcasting. In this paper, we assume that the nodes are fully synchronized as assumed in [2][3][4].

\section{Fast Flooding in Power Save Mode}

We propose a fast flooding scheme for the power save mode (FFPSM) of IEEE 802.11 DCF to reduce the end-to-end delay. Each node has to wake up periodically and stay awake for a fixed duration (ATIM Window), regardless of participating in the data transmission. If the upcoming packets can be announced to receivers of several hops ahead during the current ATIM Window, the receivers stay awake for the data transmission and the packets can move forward several hops at an interval.

This idea inevitably involves the upper layer protocols and requires the modification of the standard. The MAC protocol for wireless networks is responsible for only one-hop communication. To propagate the announcement for unicast messages through the network, the MAC frames should contain the routing information, or network layer operation should be performed at the step of ATIM exchange. In the case of broadcasting, network layer information or operation is not required because the packets have to be delivered to all nodes. Also, the broadcasting is a fundamental communication method in many essential protocols, because of the ever-changing topology in MANETs. Therefore, the proposed scheme considers only the broadcast packets by flooding, one of the simplest and reliable broadcasting algorithm [8]. We surmise that the improvement of flooding performance makes the overall network performance better and our scheme can be adapted to other broadcast algorithms.

ATIM frames in IEEE 802.11 DCF may be classified into 2 types by the destination address (DA). If DA is a group address, all single-hop neighbors which listen the announcement stay awake for the subsequent broadcast transmission. If not, the only designated node sends an ACK and stays awake, while others go to sleep. We refer to them as B-ATIM and U-ATIM for each, and introduce another class, F-ATIM. The F-ATIM contains a group address of DA and additional information to tell if it is for multi-hop broadcasting.

The ATIM and frame transmission of FFPSM is as follows. Unspecified procedures conform to the standard specification [1]. 
(a) If a mobile node has flooding packets to send, it transmits a F-ATIM frame during the ATIM Window. After the ATIM window, it remains awake and transmits the buffered flooding packets.

(b) The nodes that receive a F-ATIM frame also transmit a F-ATIM frame during the rest of the current ATIM Window and remain awake, though they don't have buffered packets yet. After they receive the flooding packet announced by the F-ATIM, they forward it to neighbors during the rest of the $B I$. If a node receives a F-ATIM frame but cannot transmit it during the current ATIM Window, the node doesn't forward the flooding packets until the beginning of next $B I$.

(c) A node transmits only one F-ATIM frame, though it receives several F-ATIMs.

(d) The node that transmits a F-ATIM shall not transmit B-ATIMs, though it has buffered broadcast packets.

(e) The nodes that receive F-ATIMs or B-ATIMs shall not transmit U-ATIMs to the originators of received ATIMs, determining that the nodes will remain awake afterwards.

We propose a tentative solution to indicate ATIM types. ATIM frame is a 802.11 management frame, of which type and subtype value in Frame Control field is 1001. U-ATIM and B-ATIM are classified by the DA field. To indicate the F-ATIM, we use the DA field combined with the Frame Body field, not used in the original ATIM frame. The value of DA field of F-ATIM is a common broadcast IP or a special multicast IP assigned by applications, and the Frame Body field contains a TTL (time-to-live) value copied from the IP header. The responsibility to set the address and TTL field is upon the applications or routing protocols in the source node. Other intermediate nodes check and copy the TTL value from the received frames. Additional use of Frame Body field is simpler than changing the definition or function of other fields, and makes the later improvement or modification of ATIM frame easier.

\section{Simulation Results and Discussion}

We simulated the proposed FFPSM under various conditions, and compared the performance in terms of end-to-end delay and power consumption with IEEE 802.11 Active mode (AM) and Power Save mode (PSM). We implemented IEEE 802.11 PSM and our FFPSM modules into the ns-2.26 network simulator[9] with wireless extensions from the CMU Monarch project[10]. Multi-hop transmission was actuated by AODV routing protocol.

A simulation area of $1000 \mathrm{~m} \times 1000 \mathrm{~m}$ is used, and we varied the number of nodes from 20 to 100, randomly placed in the area. For each number of nodes, 10 different node deployment scripts are generated and the results are averaged. We use $100 \mathrm{~ms}$ and $20 \mathrm{~ms}$ as value of BI and ATIM Window size, based on the results of [2] and [3]. To measure energy consumption, we use $1.327 \mathrm{~W}, 0.976 \mathrm{~W}$, $0.843 \mathrm{~W}$, and $0.066 \mathrm{~W}$ as value of power, consumed by the network interface in transmit, receive, idle, and doze state. 
Fig. 2 shows the results of the case of 60 nodes. 10 sources generate unicast CBR (constant bit rate) flows starting randomly between 0 and 80 sec. Each flow lasts for 20 seconds and total simulation time is $100 \mathrm{sec}$. We varied the traffic load of each flow as 10,20,30,40, and 50kbps. On the other hand, total 30 nodes are randomly chosen for flooding flows. Each node generates one flooding packet at random time.

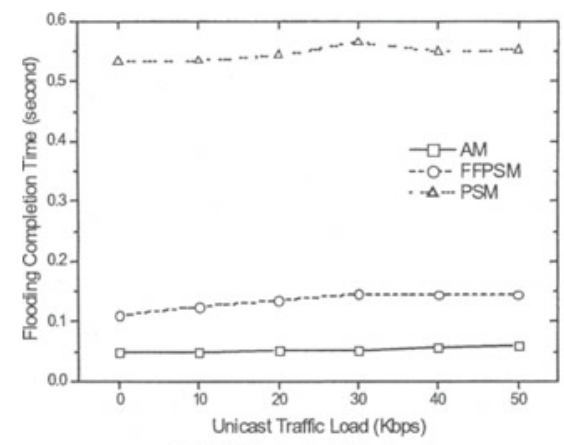

(a) FCT vs. traffic load

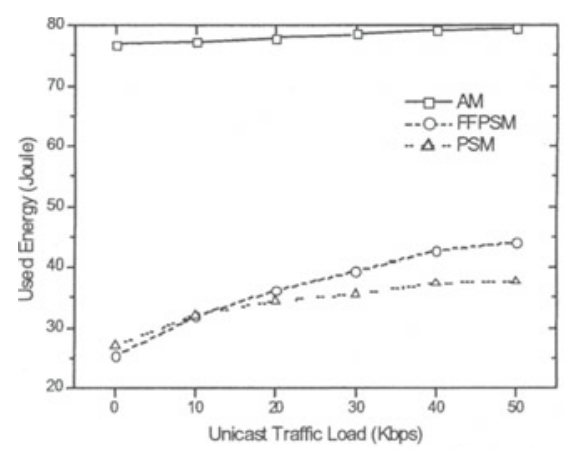

(c) energy consumption vs. traffic load

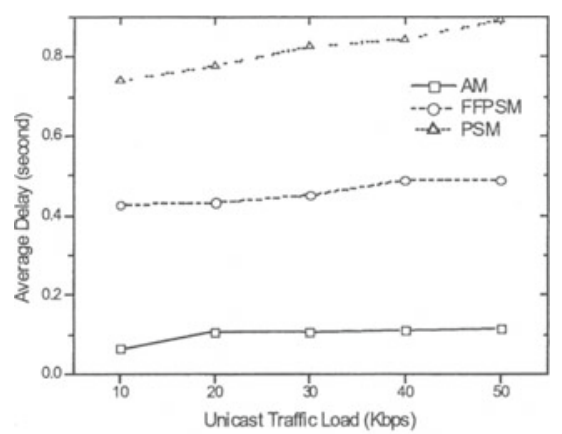

(b) unicast delay vs. traffic load

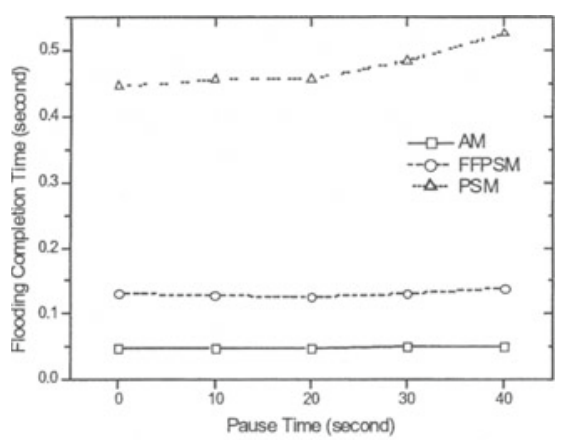

(d) FCT vs. mobility

Fig. 2. Performance comparison

In the Fig. 2(a), end-to-end delay is represented as flooding completion time (FCT), which is the time difference between the departure time from source node and the arrival time at the latest destination. FFPSM significantly improves the latency problem of PSM. In PSM, flooding packets can travel the network one hop per $B I$, so the FCT of PSM is determined by the longest hops to reach the farthest node, while FFPSM consumes just 2 BIs. FFPSM does not provide any method to reduce unicast transmission delay, but FFPSM shows better performance than PSM in unicasting, too (Fig. 2(b)). The speedup of unicast messages is explained with 2 reasons: 1) by multi-hop announcement, many nodes are awake in the same $B I$, and 2) routing protocol also uses flooding to discovery routes. 
Fig. 2(c) shows that FFPSM consumes similar amount of energy with PSM, though its transmission latency is much smaller than that of PSM.

We also evaluated the effect of mobility on the performance of FFPSM and others (Fig. 2(d)). The random waypoint model parameterized by pause time was used for node mobility. The mobility varies from constant ( 0 pause time) to low (40 pause time), and the maximum node speed is $10 \mathrm{~m} / \mathrm{s}$. The FCT values are almost same except that the FCT of PSM is more sensitive to the level of mobility.

\section{Conclusion}

In this paper, we presented Fast Flooding in Power Save Mode (FFPSM), a modification of IEEE 802.11 power save mode operation. Power saving mechanisms can reduce the power consumption, but they suffer from long latency of packet delivery due to the periodic sleeping and intermittent packet forwarding. FFPSM does not reduce the duration of the awake state for energy efficiency, but maximizes its utilization. Each node propagates the announcements of next flooding to nodes of several hops ahead, thus the packets can travel multiple hops during one beacon interval.

We evaluated the performance by simulation in terms of end-to-end delay and power consumption compared with IEEE 802.11 PSM. Simulation results showed that the proposed approach significantly reduces the end-to-end delay of flooding. For various traffic load and mobility, FFPSM showed much less delay of both flooding and unicast, with similar level of energy consumption.

\section{References}

1. IEEE Computer Society LAN MAN Standards Committee: IEEE Std 802.11-1999, Wireless LAN Medium Access Control and Physical Layer specifications (1999)

2. Woesner, H. et al.: Power-Saving Mechanisms in Emerging Standards for Wireless LANs: The MAC Level Perspective. IEEE Personal Communication (1998)

3. Jung, E.-S., Vaidya, N. H.: An Energy Efficient MAC Protocol for Wireless LANs. IEEE Infocom'02, (2002)

4. Choi, J.-M., Ko, Y.-B., Kim, J.-H.: Enhanced Power Saving Scheme for IEEE 802.11 DCF based Wireless Networks. IFIP PWC 2003, Venice, Italy (2003)

5. Chen, B. et al.: Span: An Energy-Efficient Coordination Algorithm for Topology Maintenance in Ad Hoc Wireless Networks. ACM MobiCom'01 (2001)

6. Tseng, Y.-C., Hsu, C.-S. and Hsieh, T.-Y.: Power-Saving Protocols for IEEE 802.11-Based Multi-Hop Ad Hoc Networks. IEEE Infocom'02 (2002)

7. Zheng, R., Hou, J. C., and Sha, L.: Asynchronous Wakeup for Ad Hoc Networks. ACM MobiHoc'03 (2003)

8. Williams, B., Camp, T.: Comparison of Broadcasting Techniques for Mobile Ad Hoc Networks. ACM MobiHoc'02 (2002)

9. http://www.isi.edu/nsnam/ns/index.html

10. http://www.monarch.cs.cmu.edu/cmu-ns.html 